\title{
REM sleep deprivation and drinking in rats: A test of Vogel's theory
}

\author{
ROBERT A. HICKS, STEVEN GOMEZ, MARGE GONZALES, \\ SUZANNE MCTIGHE, and DAVID ORTIZ \\ San Jose State University, San Jose, California
}

\begin{abstract}
As a further test of the hypothesis that REM sleep plays a specific role in energizing primarydrive-linked behaviors, the effects of REM sleep deprivation on the drinking behavior of rats was measured on three occasions subsequent to treatment, that is, after $1 \mathrm{~h}, 4$ days, and 8 days of recovery. Contrary to the prediction, there were no significant differences between the REMdeprived and control groups on any of the posttreatment drinking tests. Thus, these data question the validity of the REM-sleep/primary-drive hypothesis.
\end{abstract}

To explain his finding that REM sleep deprivation (RSD) improved endogenous depression, Vogel (1979) proposed a motivational theory of REM sleep (RS). In formulating this theory, he stated that this "motivational theory is not intended to preempt other RS theories ... it is simply a theory about one of several RS functions", (p. 233). As Horne and McGrath (1984) noted in their recent review, much of the evidence that could be used to buttress this theory was derived from observations of laboratory animals that suggest that RSD increased certain primary-drive-reducing behaviors, namely, eating (Bergmann, Kushida, Hennessy, Winter, \& Rechtschaffen, 1984; Bowersox, Baker, \& Dement, 1984; Dement, 1965; Elomaa \& Johansson, 1980; Siegel, 1975) and sexual activity (Morden, Mullin, Levine, Cohen, \& Dement, 1968).

Essentially, Vogel's theory suggests that the level of generalized drive is inversely related to the amount of RS or, conversely, that the drive level is a direct function of the degree of RSD. In this regard, Vogel (1979) stated that "a motivational function of RS is inferred from these observations that RSD increases drive motivated behavior. Since RS reverses the effect of RSD, it follows-and this is the crux of the motivational theory-that RS decreases waking drive motivated behavior"' (p. 234). Although the primary-drive-related evidence for this theory is relatively sparse, it appears to support the theory fairly consistently. To elaborate, in an attempt to determine the relationship between RS and spontaneous variations in food intake, Siegel (1975) continuously monitored certain sleep and food-intake parameters of 6 undisturbed ad-lib-fed cats for from 5 to 9 days. As a result of these observations, Siegel concluded that "in all but one of the cats, the correlation between REM sleep and food intake was negative. REM sleep was a better predictor of food intake than either waking, slow wave sleep or previous food intake"

This research was supported by NIH-MBRS Grant RR 08192-07. Reprint requests should be addressed to Robert A. Hicks, Department of Psychology, San Jose State University, San Jose, CA 95192. (p. 399). Recently, however, Bowersox et al. (1984) failed to replicate Siegel's research. Using a research protocol that closely approximated Siegel's, they computed negative correlations between RS and subsequent food intake for 3 of their 5 animals, and none of these were significant. As a consequence they concluded that "this study found no consistent evidence that in the undisturbed animal, food consumption is related quantitatively to daytime/nighttime expression of any sleep or waking variable' (p. 909). Thus, the possibility of a relationship between spontaneous variations in RS and subsequent food intake remains an open question.

Other researchers have attempted to establish a relationship between RS and eating by measuring the effects of RSD on subsequent food intake. The seminal study in this group (Dement, 1965) was presented as a paper at a meeting of the American Psychiatric Association, and the only published record of that research is contained in the following sentences.

Seventeen cats had their food intake carefully measured. By comparison with control cats, there was only some slight trend for the deprived cats to eat more. However, the reverse procedure (i.e., starvation) was attempted, and the motivation to eat was strikingly increased in REM-deprived cats as opposed to controls. (p. 406)

Two subsequent studies have provided less ambiguous evidence that RSD increases food intake. In one of these, Elomaa and Johansson (1980) continuously monitored the eating behaviors and the food intake of 8 male rats in an own-control design. Comparisons between the baseline and RSD periods indicated that during RSD meal patterns were altered (animals ate more frequently) and food intake increased by $32 \%$. More recently, Bergmann et al. (1984) subjected 4 rats to RSD until they died $(M=$ 39 days). During treatment, food intake increased $85 \%$ for the RSD group and 19\% for the yoked control group. They also reported that during the treatment period, the body weight of the RSD animals decreased by $32 \%$ compared with $3 \%$ for the controls. To explain these differences, they speculated that RSD greatly increased meta- 
bolic rate. Congruent with this assumption, it was reported recently (Martinez, Mendieta, \& Hicks, 1987) that a 4day period of RSD produced a $25 \%$ increase in the metabolic rate of a group of rats compared with that of the untreated controls. Collectively, then, these studies suggest that RSD increases metabolic rate and, as a consequence, food intake. Therefore, these data do not provide convincing evidence for Vogel's (1979) hyypothesis that RSD has a general activating effect on primarydrive-linked behaviors. Crucial to the validation of this general hypothesis is the unambiguous demonstration that RSD potentiates other primary-drive-linked behaviors and, in this regard, the available evidence is clearly deficient.

In the only study of its kind, Morden et al. (1968) reported in the abstract of a paper, which was presented at a meeting of the Association for the Psychophysiological Study of Sleep, that RSD increased the copulation rate of male rats that were described on the basis of an unspecified pretreatment test as being "low-copulators." In concluding their terse report of this research, Morden et al. concluded that the RSD group "showed significant change, a 'permanent' increase in sexual activity to a 'medium-high' copulation level' (p. 242). To our knowledge, this study has not been replicated, and a full report of it has never been published. Thus, when taken together, the data that can be cited to support Vogel's (1979) RS/drive hypothesis are not convincing. Therefore, the purpose of this study was to measure the relationship between RSD and another, as yet untested, primary-drive-linked behavior: drinking. Consistent with Vogel's hypothesis, we predicted that RSD would increase drinking.

\section{METHOD}

\section{Subjects}

The subjects were 20 male Sprague-Dawley rats that were 60 days old at the start of the treatment period. These animals were pretested for drinking and then were assigned to one of two groups so that each group $(n=10)$ was roughly equivalent in pretreatment level of drinking. During treatment, one of these groups was deprived of REM sleep (RSD), whereas the other group served as the large-platform controls. Throughout the experiment, these animals were maintained on a 12:12-h light:dark cycle (with lights on at $0700 \mathrm{~h}$ ) and were housed in a room with a constant temperature of $22^{\circ} \mathrm{C}$. With the exception of the 12 -h periods of water deprivation that preceded each drinking test, food and water were available continuously.

\section{REM Deprivation}

RSD was achieved using a version of the water-tank procedure modified for rats. Our RSD apparatus has been described in detail elsewhere (e.g., Hicks \& Moore, 1979). During the treatment period, all of the animals were confined in the RSD apparatus (i.e., modified 18.9-liter buckets) for a 4-day period. Each animal in the RSD group spent this time on a small platform (diameter $=6.5 \mathrm{~cm}$ ) that was surrounded by water, and thus, because of the loss of muscle tonus that accompanies RS, they were REM deprived. The control group spent the treatment period on larger platforms (diameter $=16.5 \mathrm{~cm}$ ) that were large enough to preclude contact with the water at the onset of RS and thus acted as a non-REM-deprived control. Here it should be noted that in many previous studies, it has been our practice to use both large-platform and dry-chamber control groups. Although we have never observed any difference in behavior between these control groups, we felt that, in the case of this study, the inclusion of a dry-chamber control group might have introduced a systematic bias into the data, because this group would have had less exposure to water than either RSD or control groups, thus creating the possibility of elevated thirst in this group during the first posttreatment test.

\section{Drinking Behavior}

During the 10-day adaptation period, the animals were exposed in their home cages to the Coulbourn lickometer apparatus so that they could learn to drink from it. Just prior to the treatment period, these animals were deprived of water for $12 \mathrm{~h}$ and then were given the 20min baseline drinking test in their home cages. Their responses during this test were recorded automatically by the Coulbourn lickometer. In addition to serving as a basis for equating the two groups, the baseline score for each animal was used to compute a drinking difference score for each animal on each posttreatment test. Subsequent to treatment, each animal was given a series of three 20-min drinking tests, that is, $1 \mathrm{~h}, 4$ days, and 8 days after treatment. Like the baseline test, all of these tests were carried out in the animals' home cages after a 12-h period of water deprivation. Throughout the experiment, all of the drinking tests were conducted between 0900 and $1200 \mathrm{~h}$, and responses were recorded automatically by the apparatus.

\section{Procedure}

After the animals arrived from the supplier, each animal was held for a 10-min period each day for 10 days. During this adaptation period, each animal was exposed daily to the Coulbourn lickometer apparatus for $20 \mathrm{~min}$ in its home cage. Just prior to treatment, each animal was given the baseline drinking test and then assigned to either the RSD or the control treatment group. After the 4-day treatment period, all animals were removed from the REM deprivation apparatus, towel dried, and allowed to rest for $1 \mathrm{~h}$ in their home cages. Then each animal was given the first posttreatment drinking test. Subsequent to this, drinking tests were conducted after 4 and 8 days of recovery from treatment.

It should be noted that the 12-h period of water deprivation prior to the first posttreatment test presented a problem. Prior to all of the drinking tests, water deprivation was achieved by removing the animal's water bottle at the prescribed time. However, prior to the first posttreatment test, the animals in both groups had the water surrounding their platforms available during the first $1.1 \mathrm{~h}$ of the water deprivation period. In an earlier study (Sutton, Tomlinson, \& Hicks, 1982) that required water deprivation in the RSD apparatus, we solved this problem by adulterating the water that surrounded the platforms with quinine. This is an expensive procedure. In pilot work for this study, we found that Listerine works even better than quinine so we used Listerine to adulterate the water in the RSD chambers prior to the first posttreatment drinking test.

\section{RESULTS AND DISCUSSION}

To prepare the data for each animal for analysis, a set of difference scores were computed by subtracting the animal's baseline drinking test score from each of the posttreatment drinking test scores. Then these values were transformed to standard scores $(M=50 \pm 10)$. The means and standard deviations that were computed from the standardized difference scores for both groups for each posttreatment drinking test are listed in Table 1.

The data that are summarized in Table 1 were analyzed by computing a 2 (treatment) $\times 3$ (tests) factor ANOVA with repeated measures in the second factor (tests). This analysis revealed that the main effects for both treatments and tests and the treatments $\times$ tests interaction were not significant $(F=.67, F=2.87$, and $F=1.58$, respectively). Furthermore, we tested the difference between 
Table 1

Mean Standardized Drinking Scores, Standard Deviations for

Each Treatment for Each Posttreatment Drinking Test, and the ts Between the Groups for Each Test

\begin{tabular}{lccc}
\hline \multirow{2}{*}{$\begin{array}{l}\text { Treatment } \\
\text { Group }\end{array}$} & \multicolumn{3}{c}{ Posttreatment Test } \\
\cline { 2 - 4 } REM-Deprived & $55.6 \pm 12.3$ & $51.5 \pm 12.0$ & 4-day \\
Control & $50.2 \pm 4.7$ & $48.0 \pm 7.5$ & $47.5 \pm 13.0$ \\
$t$ & $1.29 *$ & $.70^{*}$ & $.00^{*}$ \\
\hline
\end{tabular}

*Not significant.

the group means for each posttreatment drinking test and found, as is shown in Table 1, that none of these was significant. Thus, although the pattern of the posttreatment test means is congruent with the prediction made from Vogel's (1979) RS-drive hypothesis, the mean differences noted in Table 1 are not reliable and our data do not support the contention that RS plays a specific role in the modulating level of all primary-drive-linked behaviors.

We do not wish to convey the impression that our data question Vogel's (1979) assertion that RS plays a role in regulating the level of generalized drive. We do not feel that these data are directly relevant to that issue. However, we do feel that our data question the validity of the suggestion that RS is implicated, specifically, in the control of primary-drive-linked behaviors. It seems clear that when the evidence relevant to this hypothesis is considered together, these data, at best, provide weak and ambiguous support for it.

\section{REFERENCES}

Bergmann, B., Kushida, C., Hennessy, C., Winter, J., \& RechtSCHAFFEN, A. (1984). Paradoxical sleep deprivation in the rat: II. Energy consumption. Sleep Research, 13, 185.

Bowersox, S. S., Baker, T. L., \& Dement, W. C. (1984). Rapid eye movement sleep and its relationship to feeding behavior in the adult cat. Physiology \& Behavior, 92, 378-381.

DEMENT, W. C. (1965). Recent studies on the biological role of REM sleep. American Journal of Psychiatry, 122, 404-408.

ElomaA, E., \& Johansson, G. G. (1980). Rapid eye movement stage of sleep participates in the generation of the nocturnal meal pattern in the rat. Physiology \& Behavior, 24, 331-336.

HiCKs, R. A., \& MOORE, J. D. (1979). REM sleep deprivation diminishes fear in rats. Physiology \& Behavior, 22, 689-692.

Horne, J. A., \& McGrath, M. J. (1984). The consolidation hypothesis for REM sleep function: Stress and other confounding factors-A review. Biological Psychology, 18, 165-184.

Martinez, R., Mendieta, E., \& Hicks, R. A. (1987, April). REM sleep deprivation increases the metabolic rate of rats. Paper presented at the meeting of the Western Psychological Association, Long Beach, CA.

Morden, B., Mullin, R., Levine, S., Cohen, H., \& Dement, W. (1968). Effect of REM deprivation on the mating behavior of male rats. Psychophysiology, 5, 241-242.

SIEGEL, J. M. (1975). REM sleep predicts subsequent food intake. Physiology \& Behavior, 15, 399-403.

Sutton, R. L., Tomlinson, W., \& Hicks, R. A. (1982). Preacquisition REM sleep deprivation inhibits the formation of conditioned feeding suppression in rats. Bulletin of the Psychonomic Society, 20, 308-310.

VoGEL, G. W. (1979). A motivational function of REM sleep. In R. Drucker-Colin, M. Shkurovich, \& M. D. Sterman (Eds.), The functions of sleep (pp. 233-250). New York: Academic Press.

(Manuscript received for publication July 7, 1987.) 